60

The open
Boumal
loural

REVIEW ARTICLE

\title{
Urinary Cotinine as a Biomarker of Cigarette Smoke Exposure: A Method to Differentiate Among Active, Second-Hand, and Non-Smoker Circumstances
}

Andréia G.O. Fernandes ${ }^{1}$, Leonardo N. Santos ${ }^{2}$, Gabriela P. Pinheiro ${ }^{1}$, Diego da Silva Vasconcellos ${ }^{3}$, Sérgio Telles de Oliva $^{3}$, Bruno J.D. Fernandes ${ }^{4}$ and Ricardo D. Couto,"*

${ }^{1}$ Program for Asthma Control in Bahia (ProAR) and Graduate Studies in Medicine and Health, Bahia Medical School, Federal University of Bahia UFBA, Salvador, Bahia, Brazil

${ }^{2}$ The Graduate Program in Immunology, Institute of Health Sciences, Federal University of Bahia - UFBA, Salvador, Bahia, Brazil

${ }^{3}$ Environmental Analytical Chemistry Laboratory, Department of Analytical Chemistry, Institute of Chemistry, Federal University of Bahia - UFBA, Salvador, Bahia, Brazil.

${ }^{4}$ Graduate Program in Pharmacy (PPGFAR-UFBA), Clinical Biochemistry and Lipid Metabolism Laboratory, Department of Clinical Chemistry and Toxicological Analyses, Faculty of Pharmacy, Federal University of Bahia - UFBA. Barão de Jeremoabo, Salvador, Bahia, Brazil

\footnotetext{
Abstract:

Objective:

To review the literature on the use of urinary cotinine as a biological marker of cigarette smoke exposure.

Methods:

Narrative review of original and review articles on the topic of interest, published in Portuguese or English by June 2018, and selected in the following online databases: PubMed and Virtual Health Library (VHL).

Results:

Urinary cotinine is usually the recommended biomarker to estimate exposure to cigarette smoke, and can be used alone or, preferably, in association with questionnaires. Different analytical techniques can be used to quantify urinary cotinine and are differently performed because of urine sample interfering factors.

Conclusion:

The precise classification of smoking status is essential. It is advisable to use objective measurements regarding smoking habits since self-reported smoking may not always represent the true smoking status of the individual, particularly in groups that are more vulnerable to omitting the information of questionnaries, in addition, it has possible biases of memory. The accurate assessment of smoking is crucial to improve clinical management and counseling for different diseases as well as the establishment of preventive strategies. So, the use of urinary cotinine as a biomarker of cigarette smoke exposure seems to be a suitable assay to distinguish non-smokers from passive and active smokers.
}

Keywords: Biomarkers, Cotinine, Smoking, Exposure, Tobacco Smoke Pollution, Environmental Biomarkers.

Article History Received: March 17, 2020

Revised: May 21, 2020

Accepted: May 22, 2020

\section{INTRODUCTION}

Cigarette smoke exposure is one of the main risk factors associated with a marked increase in the risk of developing

* Address correspondence to this author at the Graduate Program in Pharmacy (PPGFAR-UFBA), Clinical Biochemistry and Lipid Metabolism Laboratory, Department of Clinical Chemistry and Toxicological Analyses, Faculty of Pharmacy, Federal University of Bahia/UFBA. Barão de Jeremoabo Street, 147, Ondina. Postal Code 40.170-115; Salvador, Bahia, Brazil

Tel:55 71 3283-6952/6900; E-mail: rdc@ufba.br noncommunicable diseases, cardiovascular diseases, respiratory diseases and cancers [1]. It also translates into economic costs for patients, companies, and society as a whole. These may be direct, health care-related costs, or indirect, associated with a loss of productivity [2]. The prevalence of smoking has reduced in Brazil, going from 15 to $13 \%$ between 2011 and $2013[3,4]$. According to data from the Surveillance System for Risk and Protective Factors for Chronic Diseases by Telephone Survey (VIGITEL), in 27 Brazilian capitals, the 
percentage of adult smokers in the year 2017 ranged from 4.1 in Salvador to $15.6 \%$ in Curitiba [5].

Cigarette smoke is a heterogeneous mixture of gases and particulate matter composed of more than 7000 substances, although nicotine (i.e. a tertiary amine composed of a pyridine and a pyrrolidine) stands out as the main compound present in the tobacco leaf $[6,7]$, it has a relatively short half-life when compared the cotinine [8]. Cotinine is the main metabolite of nicotine biotransformation [9]. In brief, the CYP 2A6 transforms nicotine into a nicotine-delta-iminiun ion, followed by the enzyme aldehyde oxidase action to produce cotinine, and possibly four other cotinine metabolites, such as cotinine n'- oxide, trans-3'-hidroxy-cotinine, 5' -hidroxy-cotinine, and norcotinine [9]

Cotinine concentration is proportional to the degree of exposure to nicotine and can be measured in different body fluids, such as blood, urine, and saliva, as well as in nails and hair [10]. Among these, urine is the most suitable biological fluid to detect current and secondhand smoke exposure through the quantification of cotinine. Even in situations of low exposure, the use of urine proves appropriate due to the possibility of estimating recent exposure and of showing higher concentrations, thus facilitating the use of different analytical techniques $[10,11]$. The ideal time for measurement is 4 to 8 hours after exposure, at which point the maximum levels of this biomarker can be observed [12].

In addition, cotinine concentration can be calculated directly or corrected by urinary creatinine concentration to make this biomarker method even more accurate by normalizing the results through urine dilution [11]. Although multiple measurements reduce the incidence of classification errors, a single measurement of this biomarker can accurately determine the level of exposure to tobacco smoke [13].

Determining urinary cotinine concentration has been recommended in several situations, such as monitoring of cigarette smoke exposure, even during pregnancy and in some risk groups [7, 14]; impact assessment of smoking cessation programs [15]; occupational exposure assessment [16] and; exposure to environmental pollutants [17]. Therefore, this study aimed to review the literature on the use of urinary cotinine as a biomarker of cigarette smoke exposure and the methods used for its quantification.

\section{METHODS}

A narrative review of the literature was carried out by using the following online databases: PUBMED and Virtual Health Library (VHL), which includes LILACS, IBECS, MEDLINE, Cochrane Library and SciELO. The search was conducted between April and June 2018, based on the combination of selected keywords and the Boolean Operators "AND" and "OR".

Original and review articles on the subject, in Portuguese and in English, available in full version and published by June 2018, were selected by using the following keywords: biomarkers, cotinine, smoking, and exposure. The references of the articles were also checked in order to locate those that could not be found in the databases.

\section{RESULTS AND DISCUSSION}

\subsection{Assessment of Tobacco Exposure: Urinary Cotinine Versus Self-Report}

Self-reported smoking using questionnaires has been the most widely used tool to assess exposure to cigarette smoke, however, this strategy may underestimate smoking habits. Some authors suggest that self-reporting should be associated with the analysis of specific biomarkers, especially in groups which are more likely to omit information $[18,19]$.

Markers of tobacco smoke exposure allow an estimation of the degree of exposure to cigarette smoke. In this scenario, cotinine is the main metabolite of nicotine and, therefore, largely used as a biological marker of exposure. However, the analysis of biomarkers, including urinary cotinine, depends on information obtained through self-report, which is used as a reference for the estimation of cutoff values that help define smoking status [20]. Although some cutoff values for urinary cotinine are more commonly adopted in the literature (Table 1), there is no consensus and some authors suggest that these values should be specific for each population [21].

In fact, active smokers show high levels of urinary cotinine, and the cutoff points described in the literature would be adequate for their identification. On the other hand, the differences between the other groups (i.e. exposure to secondhand smoke and non-smokers) are less clear and require different strategies to estimate cutoff points based on the data obtained by self-report and, therefore, to define more appropriate values to such populations [22, 23].

Self-report questionnaires are the main form of smoking assessment among pregnant women, however, confirmation by laboratory analysis allows correct and reliable classification [24]. In addition, double-monitoring strategies using questionnaires and urinary cotinine quantification have been used to obtain information on the smoking status and the exposure to cigarette smoke from different sources among various population groups, such as pregnant women, university students and renal transplant recipients [21, 25, 26]

In pregnant women, smoking concealment is frequent due to the influence of social factors. The same is true for patients suffering from diseases with a strong correlation with smoking, such as chronic obstructive pulmonary disease, and head and neck cancer. In these cases, self-reported smoking had no correlation with the concentration of carcinogenic metabolites, unlike urinary cotinine $[18,27,28]$. In children, secondhand smoke exposure estimates are usually obtained through the self-report of their parents or caregivers, who are likely to be the source of exposure. Therefore, accurate and objective measurements are crucial, with the use of urinary cotinine concentration being a noninvasive option widely described in the literature for this age group [29].

Also, assessing cigarette smoke exposure using both urinary cotinine quantification and questionnaires in early life allows us to estimate the risk of recurrent wheezing and asthma in childhood. In addition, smoke exposure is closely related to the greater presence of daily symptoms of asthma and its assessment helps in the identification of children at higher risk 
of experiencing an asthma crisis aggravation [29 - 31].

It is recognized that cotinine is the better predictor of birth weight than self-reported per-day tobacco use [32]. Most studies of reproductive consequences that were based on cotinine body fluid levels such as urine of mother or neonate demonstrate a better correlation between higher cotinine level and poor neonatal outcome [33, 34].

In all of these cases, the analysis of tobacco biomarkers is an indispensable tool that can be used independently to measure the exposure to cigarette smoke or, preferably, together with questionnaires.

\subsection{Urinary Cotinine for Measuring Exposure to Secondhand Smoke}

Exposure to secondhand smoke is defined as the exposure to the smoke that comes from the direct burning of cigarettes or other tobacco products, usually in combination with the smoke exhaled by the smoker, with harmful effects on the health of the exposed individual. The exposure of non-smokers to cigarette smoke depends on some factors, such as the room ventilation rate, the proximity of smokers to non-smokers, number of cigarettes smoked, among others [2]. For example, in seamen volunteers recruited from submarines, it was observed that the urinary cotinine levels of non-smoker on board were 2.1 times higher than at the seaport [35].

Cotinine is also the biomarker of choice for the quantification of exposure to secondhand smoke. It is possible to establish a dose-response relationship between the intensity and duration of exposure and the amount of cotinine excreted in the urine [36] since urinary cotinine is highly correlated with active and passive smoking [37]. Some authors believe that urinary cotinine concentration is a useful biomarker to distinguish non-smokers from current smokers. However, a careful interpretation of the cotinine concentration is necessary to estimate passive exposure to cigarette smoke [14].

The effects of secondhand smoke on children can be seen through respiratory diseases, infections, reduced school performance, and neurobehavioral problems [38]. Therefore, more effective strategies should be implemented towards protecting this population, which represents the most susceptible group to the harmful effects of environmental tobacco smoke exposure [38]. Urinary cotinine levels in children tend to vary depending on the number of household smokers or involved in their daily activities, the parents' perception of the tobacco exposure effects on children, the family members number of cigarettes smoked per day, and the exposure duration at home [39-41].

Just as it is the case for children, bar and restaurant staff are also a vulnerable population when it comes to secondhand smoke exposure. Promoting smoking cessation programs and occupational rules regarding smoking prohibition can have a significant impact on public health, and the measurement of urinary cotinine can be a valuable form of biological monitoring. In an experimental study with bar staff after the implementation of anti-smoking laws, there was a significant reduction in mean urinary cotinine concentration, from $35.9 \mathrm{ng}$ $/ \mathrm{mL}$ to below the limit of quantification $(5 \mathrm{ng} / \mathrm{mL})$, as well as in self-reported respiratory symptoms [42]. Therefore, it is clear that measures focused on occupational health such as the implementation of policies for smoke-free places are extremely relevant.

\subsection{Analytical Methodologies for Determination of Urinary Cotinine}

Urinary cotinine can be quantified by several analytical techniques, such as High- Performance Liquid Chromatography (HPLC); Gas Chromatography (GC); thinlayer chromatography; Enzyme-Linked Immunosorbent Assay (ELISA); chemiluminescent immunoassay; radioimmunoassay. Mass spectrometry or ultraviolet absorption detectors have been widely applied for the detection of cotinine in chromatographic techniques (Table 1).

Despite their high sensitivity, the immunoassays specificity is low for cotinine quantification due to cross-reactions with other nicotine metabolites, such as 3-hydroxycotinine and 3hydroxycotinine glucuronide; on the other hand, their acquisition and operating costs are lower and they can be very useful as a screening assay, especially when used in new high throughput systems, which can be highly efficient $[25,43]$. In addition, immunoassays can also be complementary to the analyses conducted with chromatographic techniques, helping achieve greater selectivity when required [26].

Chromatographic techniques are primarily separation methods with high analytical specificity as they are able to separate structurally related metabolites from nicotine. In addition, their high sensitivity allows the limit of quantification for cotinine to be as low as $0.05 \mathrm{ng} / \mathrm{mL}$ when using liquid chromatography-mass spectrometry [12]. Chromatographybased methods can selectively quantitate free cotinine in urine. Some authors have also been performing cotinine-Nglucuronide hydrolysis using alkaline or enzymatic hydrolysis in order to determine total cotinine (i.e. free and conjugated) [29, 37].

However, different chromatographic techniques such as thin-layer chromatography, liquid chromatography, and gas chromatography can be used to detect cotinine; as a limitation, to detect cotinine these methods are more expensive and timeconsuming. Usually, such techniques require urine samples prior to treatment to cotinine quantification, which may be done by purification through previous chromatography, solidphase extraction or liquid-liquid extraction [21, 23, 44].

\subsection{Variability in Urinary Cotinine Concentration}

Urine cotinine levels tend to be influenced by environmental factors related to the intensity and duration of exposure to tobacco smoke, the amount of nicotine in the cigarette, the size and ventilation of the place of exposure.

Several factors influence the metabolism of nicotine, such as ethnic differences, Black and Asian individuals have a lower nicotine metabolism rate when compared to White people [45]; dietary habits, because some types of food have nicotine in their composition, which may increase the cotinine metabolite levels [46]; age, newborns have prolonged elimination of nicotine, but similar elimination of cotinine and other 
conjugated metabolites. This may be caused by the difference in the action of the CYP2A6 enzyme, which is responsible for the metabolism of these substances [47]. Moreover, the elderly tend to have reduced renal clearance of cotinine compared to adults [48], and during pregnancy, metabolic clearance of cotinine is markedly accelerated, resulting in a shorter half-life when compared to non-pregnant women [49]. On the other hand, individuals with severe renal impairment have reduced metabolic clearance of nicotine by about $50 \%$ when compared to healthy subjects [50].

Table 1. Studies using urinary cotinine as a biomarker of tobacco smoke exposure, published in the last five years.

\begin{tabular}{|c|c|c|c|c|c|}
\hline Author, (year) & Study Design & Study Population & Level of Exposure & Analytical Method & $\begin{array}{c}\text { Urinary Cotinine } \\
\text { Concentration }\end{array}$ \\
\hline Paci, et al., 2018 & $\begin{array}{l}\text { Cross-sectional } \\
\text { study }\end{array}$ & 1,075 individuals & Smokers: $27.5 \%$ & HPLC-MS & $\begin{array}{c}\text { Cutoff point: } 100 \mu \mathrm{g} / \mathrm{g} \\
\text { creat. } \\
\text { Median - smokers: } \\
1,504.7 \text { ug/g creat. } \\
\text { Median - non-smokers: } \\
5.6 \mathrm{ug} / \mathrm{g} \text { creat. }\end{array}$ \\
\hline Perry et al., 2018 & Case-control study & 295 individuals & $\begin{array}{l}\text { Urinary cotinine was } \\
\text { detected in } 60 \text { children } \\
\text { subject to exposure at } \\
\text { home (parents' self- } \\
\text { report) and } 14 \text { children } \\
\text { whose parents denied } \\
\text { exposure. }\end{array}$ & MS & Cutoff point: $>5 \mu \mathrm{g} / \mathrm{L}$ \\
\hline Moon et al., 2017 & $\begin{array}{l}\text { Cross-sectional } \\
\text { study }\end{array}$ & $\begin{array}{c}276 \text { employees at tobacco } \\
\text { and hookah smoking } \\
\text { places }\end{array}$ & \begin{tabular}{|c|} 
Median creatinine \\
concentration \\
(interquartile): $1.1(0.2-$ \\
40.9) $\mu \mathrm{g} / \mathrm{g}$.
\end{tabular} & \begin{tabular}{|c|} 
Enzyme-linked \\
immunosorbent assay \\
kit.
\end{tabular} & $\begin{array}{l}\text { Limit of detection: } 2 \\
\text { mg/dL. }\end{array}$ \\
\hline Kim et al., 2018. & $\begin{array}{l}\text { Cross-sectional } \\
\text { study }\end{array}$ & $\begin{array}{l}96,806 \text { medical records of } \\
\text { asymptomatic individuals } \\
\text { subjected to colonoscopy }\end{array}$ & $\begin{array}{l}\text { Active smokers: } 23 \% \\
\text { Non-smokers: } 77 \%\end{array}$ & $\begin{array}{c}\text { DRI cotinine assay } \\
\text { using a } \\
\text { modular P800 } \\
\text { chemistry analyzer. }\end{array}$ & Cutoff point: $\geq 50 \mathrm{ng} / \mathrm{mL}$ \\
\hline Benowitz et al., 2018 & $\begin{array}{l}\text { Cross-sectional } \\
\text { study }\end{array}$ & 469 adolescents & $\begin{array}{l}\text { Adolescents with } \\
\text { cotinine levels above the } \\
\text { limit of quantification: } \\
407(87 \%) \text {. }\end{array}$ & LC-MS & $\begin{array}{l}\text { Limit of quantification: } \\
0.05 \mathrm{ng} / \mathrm{mL} \text {. }\end{array}$ \\
\hline Nam et al., 2017 & $\begin{array}{c}\text { Cross-sectional } \\
\text { study }\end{array}$ & $\begin{array}{c}1,139 \\
\text { adolescents }\end{array}$ & N.I. & GC-MS & $\begin{array}{c}\text { Limit of detection: } 0.26 \\
\text { ng/mL. }\end{array}$ \\
\hline $\begin{array}{l}\text { Wang, et al., } \\
(2017)\end{array}$ & $\begin{array}{l}\text { Cross-sectional } \\
\text { study }\end{array}$ & $\begin{array}{l}368 \text { children and their } \\
\text { parents }\end{array}$ & $\begin{array}{c}\text { Children living with } 2 \text { or } \\
\text { more smokers: } 30.7 \% \text {; } \\
\text { Children living with } 1 \\
\text { smoker: } 69.3 \% .\end{array}$ & GC-MS & $\begin{array}{l}\text { Geometric mean for } \\
\text { children: } 3.94 \mathrm{ng} / \mathrm{mL} \text {. }\end{array}$ \\
\hline $\begin{array}{c}\text { Martinez-Sanchez, et } \\
\text { al., } 2017\end{array}$ & $\begin{array}{l}\text { Cross-sectional } \\
\text { study }\end{array}$ & 49 non-smokers & $\begin{array}{l}\text { Individuals living with } \\
\text { smoker(s): } 25\end{array}$ & LC-MS & $\begin{array}{c}\text { Perception of intensity of } \\
\text { exposure (Median) } \\
\text { High: } 7.59 \mathrm{ng} / \mathrm{mL} ; \\
\text { Medium: } 3.57 \mathrm{ng} / \mathrm{mL} ; \\
\text { Low: } 1.25 \mathrm{ng} / \mathrm{mL} ; \\
\text { Very low: } 0.44 \mathrm{ng} / \mathrm{mL} \text {. }\end{array}$ \\
\hline Rifai, et al., 2017 & $\begin{array}{l}\text { Cross-sectional } \\
\text { study }\end{array}$ & 843 active smokers & $\begin{array}{c}1 \text { to } 10 \text { cigarettes/day: } \\
299 \\
10 \text { to } 20 \text { cigarettes/day: } \\
443 \\
>20 \text { cigarettes/day: } 101\end{array}$ & Immulite 2000 Assay & $\begin{array}{c}\text { Tercile 1: } 7 \text { - } 2421 \mathrm{ng} / \mathrm{mL} ; \\
\text { Tercile } 2: 2422-6436 \\
\text { ng/mL; } \\
\text { Tercile } 3:>6437 \mathrm{ng} / \mathrm{mL} .\end{array}$ \\
\hline $\begin{array}{l}\text { Hoseini, et al., } \\
2016\end{array}$ & $\begin{array}{l}\text { Cross-sectional } \\
\text { study }\end{array}$ & 222 urban residents & $\begin{array}{c}\text { Active smokers: } 76 \\
\text { Passive smokers: } 57 \\
\text { Non-smokers: } 89\end{array}$ & ELISA & $\begin{array}{c}\text { Cutoff point(active): } 100 \\
\text { ng/mL } \\
\text { Active smoker: } 795.6 \pm \\
396.7 \mathrm{ng} / \mathrm{mL} ; \\
\text { Passive smoker: } 7.6 \pm 2.8 \\
\text { ng/mL; } \\
\text { Non-smoker: } 3.56 \pm 1.9 \\
\text { ng/mL. }\end{array}$ \\
\hline Tranfo et al., (2016) & Descriptive study & $\begin{array}{l}446 \text { healthy volunteer } \\
\text { residents }\end{array}$ & $\begin{array}{c}\text { Smokers: } 93 \\
\text { Former smokers: } 156 \\
\text { Non-smokers: } 197\end{array}$ & HPLC-MS & $\begin{array}{c}\text { Limit of detection: } 12.41 \\
\mu \mathrm{g} / \mathrm{L} . \\
\text { Cutoff point (smokers): } \\
100 \mu \mathrm{g} / \mathrm{g} \text { creatinine. } \\
>100 \mathrm{ug} / \mathrm{g} \text { creatinine: } 110\end{array}$ \\
\hline
\end{tabular}




\begin{tabular}{|c|c|c|c|c|c|}
\hline Author, (year) & Study Design & Study Population & Level of Exposure & Analytical Method & $\begin{array}{l}\text { Urinary Cotinine } \\
\text { Concentration }\end{array}$ \\
\hline Hellemons, et al. (2015) & Prospective cohort & $\begin{array}{l}603 \text { renal transplant } \\
\text { recipients }\end{array}$ & $\begin{array}{l}\text { Never smoked: } 217 \\
\text { Former smokers: } 255 \\
\text { Light smokers: } 64 \\
\text { Heavy smokers: } 67\end{array}$ & Immulite 2500 Assay & $\begin{array}{c}\text { Limit of detection: } 10 \\
\text { ng/mL. } \\
\text { Cutoff point } \\
\text { Non-smokers: }<100 \\
\text { ng/mL; } \\
\text { Passive smokers: } \\
\text { 100-500 ng/mL; } \\
\text { Active smokers: }>500 \\
\text { ng/mL; }\end{array}$ \\
\hline $\begin{array}{l}\text { Lupsa, et al. } \\
\quad(2015)\end{array}$ & $\begin{array}{l}\text { Cross-sectional } \\
\text { study }\end{array}$ & $\begin{array}{l}360 \text { children and their } \\
\text { mothers }\end{array}$ & $\begin{array}{l}\text { Mothers } \\
\text { Daily smokers: } 89 \\
\text { Occasional smokers: } \\
\quad 30 \\
\text { Former smokers: } 62 \\
\text { Non-smokers: } 179\end{array}$ & HPLC-MS & $\begin{array}{l}\text { Limit of quantification: } \\
0.7 \mathrm{ug} / \mathrm{L} \text {. } \\
\text { Different cutoff points for } \\
\text { each subpopulation. }\end{array}$ \\
\hline $\begin{array}{l}\text { Evlampidou, et al., } \\
\text { (2015) }\end{array}$ & Cohort & $\begin{array}{l}175 \text { pairs of non-smoking } \\
\text { mothers-children }\end{array}$ & \begin{tabular}{|} 
Children with no \\
exposure to secondhand \\
smoke at 8 months \\
(mothers' self-report): \\
$56 \%$
\end{tabular} & GC-MS & $\begin{array}{l}\text { Total Cotinine (free }+ \\
\text { glucuronide) } \\
\text { Limit of detection: } 1.0 \\
\text { ng/mL. } \\
\text { Cutoff point: } 100 \mathrm{ng} / \mathrm{mL}\end{array}$ \\
\hline Mørck, et al., (2015) & $\begin{array}{l}\text { Cross-sectional } \\
\text { study }\end{array}$ & $\begin{array}{c}75 \text { pairs of } \\
\text { mothers/children from } \\
\text { urban areas; } \\
70 \text { pairs of } \\
\text { mothers/children from } \\
\text { rural areas }\end{array}$ & $\begin{array}{l}\text { Smoking mothers from } \\
\text { urban areas: } 6 \\
\text { Smoking mothers from } \\
\text { rural areas: } 12\end{array}$ & LC-MS & $\begin{array}{c}\text { Limit of detection: } 0.3 \\
\text { ug/L. } \\
\text { Children's maximum } \\
\text { value: } 16.3 \mathrm{ug} / \mathrm{L} ; \\
\text { Mothers' maximum value: } \\
3.403 \mathrm{ug} / \mathrm{L} \text {; } \\
\text { All smoking mothers: }> \\
200 \mathrm{ug} / \mathrm{L}\end{array}$ \\
\hline Wang, et al., (2015) & $\begin{array}{l}\text { Randomized } \\
\text { controlled trial }\end{array}$ & $\begin{array}{l}65 \text { children aged } 5 \text { to } 6 \\
\text { years and caregivers. } \\
33 \text { pairs received } \\
\text { intervention (smoking } \\
\text { cessation education); } \\
32 \text { control pairs. }\end{array}$ & $\begin{array}{c}\text { Cessation after } 6 \text { months } \\
\text { Intervention group: } \\
34.4 \% \text {; } \\
\text { Control group: } 0 \%\end{array}$ & GC-MS & $\begin{array}{l}\text { Limit of quantification: } \\
0.1 \mathrm{ng} / \mathrm{mL} \text {. }\end{array}$ \\
\hline $\begin{array}{l}\text { Khariwala, et al. } \\
\qquad(2015)\end{array}$ & $\begin{array}{l}\text { Cross-sectional } \\
\text { study }\end{array}$ & $\begin{array}{c}84 \text { smokers with head and } \\
\text { neck cancer }\end{array}$ & N.I. & GC-MS & $\begin{array}{c}\text { Urinary cotinine levels } \\
\text { correlated with carcinogen } \\
\text { levels. }\end{array}$ \\
\hline Stelmach, et al. (2015) & $\begin{array}{l}\text { Cross-sectional } \\
\text { study }\end{array}$ & $\begin{array}{l}144 \text { individuals with } \\
\text { Asthma (51) or Chronic } \\
\text { Obstructive Pulmonary } \\
\text { Disease (53) }\end{array}$ & $\begin{array}{c}\text { Smokers: } 20 \\
\text { Never smoked: } 20\end{array}$ & HPLC-UV & $\begin{array}{l}\text { Median concentration } \\
\text { Smokers: } 2036 \mathrm{ng} / \mathrm{mL} \text {; } \\
\text { Never smoked: } 70 \\
\text { ng/mL; } \\
\text { COPD: } 167 \mathrm{ng} / \mathrm{mL} \text {; } \\
\text { Asthma: } 47 \mathrm{ng} / \mathrm{mL} \text {. }\end{array}$ \\
\hline $\begin{array}{l}\text { Jones, et al. } \\
\quad(2014)\end{array}$ & $\begin{array}{l}\text { Experimental } \\
\text { exposure }\end{array}$ & 10 participants & $\begin{array}{l}\text { Non-smokers: } 08 \\
\text { Active smokers: } 02\end{array}$ & LC-MS & $\begin{array}{c}\text { Total Cotinine. } \\
\text { Limit of quantification: } \\
0.05 \mathrm{ng} / \mathrm{mL} \text {. }\end{array}$ \\
\hline $\begin{array}{l}\text { Khariwala, et al. } \\
\qquad(2014)\end{array}$ & Cross section & $\begin{array}{l}28 \text { black individuals, } 04 \\
\text { Latinos and } 25 \text { whites } \\
\text { from one community }\end{array}$ & $\begin{array}{c}\text { Smoked at least } 1 \\
\text { cigarette in } 4-24 \text { days in } \\
\text { the last } 30 \text { days. }\end{array}$ & LC-MS & $\begin{array}{c}\text { Limit of quantification: } \\
0.05 \mathrm{ng} / \mathrm{mL} . \\
\text { Mean (standard } \\
\text { deviation): } 804.40 \\
\text { (917.76) ng / mg } \\
\text { creatinine; Median: } 409.9 \\
\text { ng / mg creatinine. }\end{array}$ \\
\hline $\begin{array}{c}\text { Martinez-Sanchez, et } \\
\text { al., 2017 } \\
(\mathbf{2 0 1 4 )}\end{array}$ & Observational study & $\begin{array}{c}49 \text { non-smoking } \\
\text { volunteers from different } \\
\text { households }\end{array}$ & $\begin{array}{l}\text { People living with } \\
\text { smoker(s): } 25 ; \\
\text { People living in non- } \\
\text { smoking households: } \\
24 .\end{array}$ & LC-MS & $\begin{array}{l}\text { Limit of quantification: } \\
0.10 \mathrm{ng} / \mathrm{mL} \text {. } \\
\text { Median: } 0.92 \mathrm{ng} / \mathrm{mL} \text {. }\end{array}$ \\
\hline $\begin{array}{c}\text { Gill; Krishnan; Dozor, } \\
2014\end{array}$ & $\begin{array}{l}\text { Cross-sectional } \\
\text { study }\end{array}$ & $\begin{array}{l}40 \text { individuals aged } 8-18 \\
\text { years, with mild to } \\
\text { moderate persistent } \\
\text { asthma. }\end{array}$ & $\begin{array}{l}\text { Individuals affected by } \\
\text { secondhand smoke } \\
\text { exposure: } 28(70 \%)\end{array}$ & ELISA & $\begin{array}{l}\text { Indication of exposure to } \\
\text { secondhand smoke: } \geq 1 \mathrm{ng} \\
\qquad / \mathrm{mL} .\end{array}$ \\
\hline
\end{tabular}




\begin{tabular}{|c|c|c|c|c|c|}
\hline Author, (year) & Study Design & Study Population & Level of Exposure & Analytical Method & $\begin{array}{l}\text { Urinary Cotinine } \\
\text { Concentration }\end{array}$ \\
\hline $\begin{array}{l}\text { Mateos-Vílchez, et al. } \\
\text { (2014) }\end{array}$ & $\begin{array}{l}\text { Cross-sectional } \\
\text { study }\end{array}$ & $\begin{array}{l}1,813 \text { women from } 03 \\
\text { independent samples: } \\
\text { beginning, end of } \\
\text { pregnancy and immediate } \\
\text { postpartum period. }\end{array}$ & $\begin{array}{l}\text { Tobacco exposure } \\
\text { (active and passive } \\
\text { smoking) } \\
\text { End of gestation: } \\
25.0 \% \text {; } \\
\text { Beginning of } \\
\text { gestation: } 41.8 \% \text {; }\end{array}$ & $\begin{array}{l}\text { Competitive } \\
\text { chemiluminescent } \\
\text { immunoassay }\end{array}$ & $\begin{array}{c}\text { Non-smokers: }<20 \\
\text { ng/mL; } \\
\text { Passive or occasional } \\
\text { smokers: } 20-125 \mathrm{ng} / \mathrm{mL} ; \\
\text { Moderate smokers: } \\
\text { 125-500 ng / mL; } \\
\text { Heavy smokers: }>500 \mathrm{ng} \\
\text { / mL. }\end{array}$ \\
\hline Machado, et al. (2014) & $\begin{array}{l}\text { Cross-sectional } \\
\text { study }\end{array}$ & 125 pregnant women & $\begin{array}{l}\text { Current smokers: } 37 \text {; } \\
\text { Individuals subject to } \\
\text { secondhand smoke } \\
\text { exposure: } 25 \\
\text { Non-smokers: } 63\end{array}$ & HPLC-UV & $\begin{array}{l}\text { Urinary cotinine limit of } \\
\text { quantification: } 10 \mathrm{ug} / \mathrm{L} \text {. }\end{array}$ \\
\hline $\begin{array}{l}\text { Matsumoto, et al. } \\
\qquad(2013)\end{array}$ & $\begin{array}{l}\text { Cross-sectional } \\
\text { study }\end{array}$ & $\begin{array}{l}219 \text { people from a } \\
\text { manufacturing company. }\end{array}$ & $\begin{array}{c}\text { Smokers: } 102 ; \\
\text { Non-smokers: } 117\end{array}$ & GC-MS & $\begin{array}{c}\text { Limit of quantification: } \\
0.7 \mathrm{ng} / \mathrm{mL} \text {. } \\
\text { Smokers: } 3.948 \mathrm{ng} / \mathrm{mL} \text {; } \\
\text { Non-smokers: }<2.8 \\
\text { ng/mL; }\end{array}$ \\
\hline $\begin{array}{l}\text { Szumska, et al. (2013) } \\
\text { Tyrpién, et al. }(2000)\end{array}$ & $\begin{array}{l}\text { Cross-sectional } \\
\text { study }\end{array}$ & 85 medical students & $\begin{array}{l}\text { Active smokers: } 40 \\
\text { Non-smokers: } 45 \\
\text { Exposed: } 25 \\
\text { Not exposed: } 20\end{array}$ & $\begin{array}{c}\text { ELISA for nicotine } \\
\text { metabolites, followed } \\
\text { by C18 TLC with } \\
\text { densitometry }\end{array}$ & $\begin{array}{c}\text { ELISA (nicotine } \\
\text { metabolites) } \\
\text { Smokers: }>200 \mu \mathrm{g} / \mathrm{g} \\
\text { creatinine; } \\
\text { Non-smokers: }<200 \mu \mathrm{g} / \mathrm{g} \\
\text { creatinine; } \\
\text { Passive smoker: } 20-200 \\
\mu \mathrm{g} / \mathrm{g} \text { creatinine; } \\
\text { Not exposed: }<20 \mu \mathrm{g} / \mathrm{g} \\
\text { creatinine. } \\
\text { TLC } \\
\text { Limit of detection: } 13.5 \\
\text { ng/spot. } \\
\text { Smokers: } 523.1 \pm 68.1 \\
\text { ug/g creatinine; } \\
\text { Non-smokers } \\
\text { Exposed: } 40.89 \pm 24.8 \\
\mu \mathrm{g} \text { cotinine } / \mathrm{g} \text { creatinine. } \\
\text { Not exposed: not } \\
\text { detected. }\end{array}$ \\
\hline Vardavas, et al. (2013) & Cohort & $\begin{array}{l}367 \text { non-smoking } \\
\text { pregnant women }\end{array}$ & $\begin{array}{c}\text { Exposure to secondhand } \\
\text { cigarette smoke } \\
>2 \text { sources of } \\
\text { exposure: } 158 ; \\
\leq 2 \text { sources of } \\
\text { exposure: } 209\end{array}$ & LC-MS & $\begin{array}{c}\text { Total Cotinine. } \\
\text { Limit of quantification: } \\
0.5 \mathrm{ng} / \mathrm{mL} \text {. } \\
\text { Household exposure: } 4.40 \\
\mathrm{ng} / \mathrm{mL} \text { increase; } \\
\text { Secondhand smoke } \\
\text { exposure in cars: } 8.73 \mathrm{ng} / \\
\text { mL increase. } \\
\end{array}$ \\
\hline $\begin{array}{l}\text { Yarnall, et al. } \\
\quad(2013)\end{array}$ & Longitudinal study & $\begin{array}{l}239 \text { volunteers recruited } \\
\text { from US Navy } \\
\text { submarines. }\end{array}$ & $\begin{array}{c}\text { Pairs of non-smoker } \\
\text { samples at seaport } \\
\text { (before embarking) and } \\
\text { after disembarking: } 206 \\
\end{array}$ & LC-MS & $\begin{array}{l}\text { Limit of detection: } 0.05 \\
\text { ng/mL. } \\
\text { Cutoff point(smoker): } 15 \\
\text { ng/mL }\end{array}$ \\
\hline $\begin{array}{l}\text { Kim, et al. } \\
\text { (2013) }\end{array}$ & $\begin{array}{l}\text { Cross-sectional } \\
\text { study }\end{array}$ & $\begin{array}{c}925 \text { post-menopause } \\
\text { women }\end{array}$ & Never smoked & GC-MS & $\begin{array}{c}\text { Limit of detection: } 0.28 \\
\text { ng/mL. }\end{array}$ \\
\hline Pacheco, et al., (2013) & $\begin{array}{l}\text { Cross-sectional } \\
\text { study }\end{array}$ & 96 workers & $\begin{array}{c}\text { Smokers: } 26 ; \\
\text { Non-smokers: } 70 .\end{array}$ & GC-MS & $\begin{array}{c}\text { Limit of quantification: } 5 \\
\text { ng } / \mathrm{mL} \text {. }\end{array}$ \\
\hline
\end{tabular}

*Abbreviations: LC: Liquid chromatography; MS: Mass Spectrometry; GC: Gas Chromatography; ELISA: Enzyme-linked immunosorbent assay; HPLC: Highperformance liquid chromatography; UV: Ultraviolet; N.I.: No Information; ln: natural logarithm.

There is evidence that genetic polymorphisms related to nicotine metabolism constitute an important factor in the susceptibility to nicotine dependence; genetic discoveries may allow the identification of individuals at greater risk of tobacco dependence and be used as a more effective strategy in the treatment and prevention of smoking $[9,51]$. Understanding interindividual variability in nicotine metabolism is crucial, as there is substantial evidence to suggest that interindividual differences in cotinine production can be associated with CYP2A6 gene polymorphisms [52]. Japanese individuals, for example, have low CYP2A6 activity, an enzyme necessary for nicotine to be metabolized into cotinine [53].

Another important factor to estimate exposure to cigarette smoke is the establishment of cutoff points for more objective differentiation levels of exposure based on urinary cotinine 
concentration [54]; thus, factors influencing cotinine levels in urine should be considered in order to ensure better differentiation in the studied population.

\section{CONCLUSION}

Urinary cotinine is a reliable biomarker, widely used for distinguishing between active and secondhand smoke exposure. Although several highly sensitive analytical methodologies such as chromatography or immunoassay can be used for the urinary cotinine quantification, it should be preferably used in association with self-reports interviews or questionnaires, to correctly estimate the most appropriate cutoff points for smoking status classification.

\section{CONSENT FOR PUBLICATION}

Not Applicable.

\section{FUNDING}

We thank CNPq, FAPESB, and CAPES - finance Code 001, for the scholarships, financial grants, and infrastructural support.

\section{CONFLICT OF INTEREST}

The manuscript authors declare no conflict of interest. This paper was written by using secondary data information, and none of this content has been previously published.

\section{ACKNOWLEDGEMENTS}

We thank Dr. Álvaro Augusto Souza da Cruz Filho for the everlasting mentoring and friendlily teaching.

\section{REFERENCES}

[1] Ghasemian A, Rezaei N, Saeedi Moghaddam S, et al. Tobacco Smoking Status and the Contribution to Burden of Diseases in Iran, 1990-2010: findings from the Global Burden of Disease Study 2010. Arch Iran Med 2015; 18(8): 493-501. [PMID: 26265517]

[2] WHO. Protection from exposure to second-hand tobacco smoke. Policy recommendations. 2007. Available from: https://apps.who. int/iris/bitstream/handle/10665/43677/9789241563413_eng.pdf;jsess ionid=F2A13DFF6729CB01ADCC138BFC1927DC? sequence $=1$

[3] Organization WH. WHO report on the global tobacco epidemic, 2013: enforcing bans on tobacco advertising, promotion and sponsorship. Geneva: World Health Organization 2013.

[4] WHO. WHO report on the global tobacco epidemic, 2015: raising taxes on tobacco 2015.

[5] Brasil Vigitel Brasil 2016: vigilância de fatores de risco e proteção para doenças crônicas por inquérito telefônico: estimativas sobre frequência e distribuição sociodemográfica de fatores de risco e proteção para doenças crônicas nas capitais dos 26 estados b. Brasília: Ministério da Saúde 2017.

[6] Eriksen J, Schluger N, Islami F, Drope JMM. https://tobaccoatlas.org/

[7] Haufroid V, Lison D. Urinary cotinine as a tobacco-smoke exposure index: a minireview. Int Arch Occup Env Heal [Internet]. 1998;71(3):162-8. Available from: http://www.ncbi.nlm.nih.gov/ pubmed/9591157

[http://dx.doi.org/10.1007/s004200050266]

[8] Florescu A, Ferrence R, Einarson T, Selby P, Soldin O, Koren G. Methods for quantification of exposure to cigarette smoking and environmental tobacco smoke: focus on developmental toxicology. Ther Drug Monit 2009; 31(1): 14-30.http://www.ncbi.nlm.nih.gov/ pubmed/19125149

[http://dx.doi.org/10.1097/FTD.0b013e3181957a3b]

[PMID: 19125149]

[9] Tutka P, Mosiewicz J, Wielosz M. Pharmacokinetics and metabolism of nicotine. Pharmacol Rep 2005; 57(2): 143-53.http://www.ncbi.nlm. nih.gov/pubmed/15886412

[PMID: 15886412]

[10] Avila-Tang E, Al-Delaimy WK, Ashley DL, et al. Assessing secondhand smoke using biological markers. Tob Control 2013; 22(3): 164-71.http://www.ncbi.nlm.nih.gov/pubmed/22940677

[http://dx.doi.org/10.1136/tobaccocontrol-2011-050298] [PMID: 22940677]

[11] Benowitz NL, Dains KM, Dempsey D, Herrera B, Yu L, Jacob P III. Urine nicotine metabolite concentrations in relation to plasma cotinine during low-level nicotine exposure. Nicotine Tob Res 2009; 11(8): 954-60.http://www.ncbi.nlm.nih.gov/pubmed/19525206 [http://dx.doi.org/10.1093/ntr/ntp092] [PMID: 19525206]

[12] Jones IA, St Helen G, Meyers MJ, et al. Biomarkers of secondhand smoke exposure in automobiles. Tob Control 2014; 23(1): 51-7.https://www.ncbi.nlm.nih.gov/pubmed/23349229

[http://dx.doi.org/10.1136/tobaccocontrol-2012-050724] [PMID: 23349229]

[13] Lee K, Lim S, Bartell S, Hong YC. Interpersonal and temporal variability of urinary cotinine in elderly subjects. Int J Hyg Env Heal [Internet]. 2011;215(1):46-50. Available from: http://www.ncbi.nlm.nih. gov/pubmed/21900044

[http://dx.doi.org/10.1016/j.ijheh.2011.07.007]

[14] Jung S, Lee IS, Kim SB, et al. Urine Cotinine for Assessing Tobacco Smoke Exposure in Korean: Analysis of the Korea National Health and Nutrition Examination Survey (KNHANES). Tuberc Respir Dis [Internet]. 2012/10/31. 2012;73(4):210-8. Available from: https://www.ncbi.nlm.nih.gov/ pubmed/23166556

[15] Wang Y, Huang Z, Yang M, Wang F, Xiao S. Reducing environmental tobacco smoke exposure of preschool children: a randomized controlled trial of class-based health education and smoking cessation counseling for caregivers. Int $\mathrm{J}$ Env Res Public Heal [Internet]. 2015;12(1):692-709. Available from: http://www.ncbi.nlm.nih.gov/ pubmed $/ 25590146$

[http://dx.doi.org/10.3390/ijerph120100692]

[16] Pacheco SA, Torres VM, Louro H, et al. Effects of occupational exposure to tobacco smoke: is there a link between environmental exposure and disease? J Toxicol Environ Health A 2013; 76(4-5): 311-27.https://www.ncbi.nlm.nih.gov/pubmed/23514073

[http://dx.doi.org/10.1080/15287394.2013.757269] [PMID: 23514073]

[17] Sul D, Ahn R, Im H, et al. Korea National Survey for Environmental Pollutants in the human body 2008: 1-hydroxypyrene, 2-naphthol, and cotinine in urine of the Korean population. Environ Res 2012; 118 : 25-30.http://www.ncbi.nlm.nih.gov/pubmed/22939007 [http://dx.doi.org/10.1016/j.envres.2012.07.010] [PMID: 22939007]

[18] Stelmach R, Fernandes FL, Carvalho-Pinto RM, et al. Comparison between objective measures of smoking and self-reported smoking status in patients with asthma or COPD: are our patients telling us the truth? J Bras Pneumol 2015; 41(2): 124-32.http://www.ncbi.nlm.nih. gov/pubmed/25972966

[http://dx.doi.org/10.1590/S1806-37132015000004526] [PMID: 25972966]

[19] Connor Gorber S, Schofield-Hurwitz S, Hardt J, Levasseur G, Tremblay M. The accuracy of self-reported smoking: a systematic review of the relationship between self-reported and cotinine-assessed smoking status. Nicotine Tob Res 2009; 11(1): 12-24.http://www.ncbi. nlm.nih.gov/pubmed/19246437

[http://dx.doi.org/10.1093/ntr/ntn010] [PMID: 19246437]

[20] Zielińska-Danch W, Wardas W, Sobczak A, Szołtysek-Bołdys I. Estimation of urinary cotinine cut-off points distinguishing nonsmokers, passive and active smokers. Biomarkers 2007; 12(5): 484-96.https://www.ncbi.nlm.nih.gov/pubmed/17701747 [http://dx.doi.org/10.1080/13547500701421341] [PMID: 17701747]

[21] Lupsa IR, Nunes B, Ligocka D, et al. Urinary cotinine levels and environmental tobacco smoke in mothers and children of Romania, Portugal and Poland within the European human biomonitoring pilot study. Environ Res 2015; 141: 106-17.http://www.ncbi.nlm.nih. gov/pubmed $/ 25841796$

[http://dx.doi.org/10.1016/j.envres.2015.03.018] [PMID: 25841796]

[22] Hoseini M, Yunesian M, Nabizadeh R, Yaghmaeian K, Parmy S, Gharibi H, et al. Biomonitoring of tobacco smoke exposure and selfreported smoking status among general population of Tehran, Iran. Env Sci Pollut Res Int [Internet]. 2016/09/27. 2016;23(24):25065-73. Available from: https://www.ncbi.nlm.nih.gov/pubmed/27677995

[23] Mørck TA, Nielsen F, Nielsen JK, et al. The Danish contribution to the European DEMOCOPHES project: A description of cadmium, cotinine and mercury levels in Danish mother-child pairs and the 
perspectives of supplementary sampling and measurements. Environ Res 2015; 141: 96-105.https://www.ncbi.nlm.nih.gov/pubmed/ 25440293

[http://dx.doi.org/10.1016/j.envres.2014.07.028] [PMID: 25440293]

[24] Stragierowicz J, Mikołajewska K, Zawadzka-Stolarz M, Polańska K, Ligocka D. Estimation of cutoff values of cotinine in urine and saliva for pregnant women in Poland. BioMed Res Int 2013; 2013386784http://www.ncbi.nlm. nih.gov/pubmed/24228246

[http://dx.doi.org/10.1155/2013/386784] [PMID: 24228246]

[25] Hellemons ME, Sanders JS, Seelen MA, et al. Assessment of Cotinine Reveals a Dose-Dependent Effect of Smoking Exposure on Long-term Outcomes After Renal Transplantation. Transplantation 2015; 99(9): 1926-32.http://www.ncbi.nlm.nih.gov/pubmed/25710609 [http://dx.doi.org/10.1097/TP.0000000000000636] [PMID: 25710609]

[26] Szumska M, Tyrpień K, Kowalska M, Wielkoszyński T, Dobosz C. Medicine students and exposure to environmental tobacco smoke. Int $\mathrm{J}$ Occup Med Env Heal [Internet]. 2013;26(2):313-20. Available from: https://www.ncbi.nlm.nih.gov/pubmed/23771859 [http://dx.doi.org/10.2478/s13382-013-0100-8]

[27] Khariwala SS, Carmella SG, Stepanov I, et al. Self-reported Tobacco use does not correlate with carcinogen exposure in smokers with head and neck cancer. Laryngoscope 2015; 125(8): 1844-8.http://www.ncbi. nlm.nih.gov/pubmed/25877866

[http://dx.doi.org/10.1002/lary.25290] [PMID: 25877866]

[28] Mateos-Vílchez PM, Aranda-Regules JM, Díaz-Alonso G, et al. [Smoking prevalence and associated factors during pregnancy in Andalucía 2007-2012]. Rev Esp Salud Publica 2014; 88(3): 369-81.http://www.ncbi.nlm.nih.gov/pubmed/25028305 [PMID: 25028305]

[29] Butz AM, Breysse P, Rand C, Curtin-Brosnan J, Eggleston P, Diette $\mathrm{GB}$, et al. Household smoking behavior: effects on indoor air quality and health of urban children with asthma. Matern Child Heal J [Internet]. 2011;15(4):460-8. Available from https://www.ncbi.nlm. nih.gov/pubmed/ 20401688

[http://dx.doi.org/10.1007/s10995-010-0606-7]

[30] Carlsten C, Dimich-Ward H, DyBuncio A, Becker AB, Chan-Yeung M. Cotinine versus questionnaire: early-life environmental tobacco smoke exposure and incident asthma. BMC Pediatr 2012; 12 : 187.https://www.ncbi.nlm.nih.gov/pubmed/23216797 [http://dx.doi.org/10.1186/1471-2431-12-187] [PMID: 23216797]

[31] Rabinovitch N, Reisdorph N, Silveira L, Gelfand EW. Urinary leukotriene $\mathrm{E}_{4}$ levels identify children with tobacco smoke exposure at risk for asthma exacerbation. J Allergy Clin Immunol 2011; 128(2): 323-7.https://www.ncbi.nlm.nih.gov/pubmed/21807251 [http://dx.doi.org/10.1016/j.jaci.2011.05.035] [PMID: 21807251]

[32] Spector LG, Murphy SE, Wickham KM, Lindgren B, Joseph AM. Prenatal Tobacco Exposure and Cotinine in Newborn Dried Blood Spots. Pediatrics [Internet]. 2014 Jun 1 [cited 2019 Mar 31];133(6):e1632-8. Available from: http://www.ncbi.nlm.nih.gov/ pubmed/24819573

[http://dx.doi.org/10.1542/peds.2013-3118]

[33] England LJ, Kendrick JS, Gargiullo PM, Zahniser SC, Hannon WH. Measures of maternal tobacco exposure and infant birth weight at term. Am J Epidemiol [Internet]. 2001 May 15 [cited 2019 Mar 31];153(10):954-60. Available from: http://www.ncbi.nlm.nih.gov/ pubmed/11384951

[http://dx.doi.org/10.1093/aje/153.10.954]

[34] Braun JM, Daniels JL, Poole C, Olshan AF, Hornung R, Bernert JT, et al. A prospective cohort study of biomarkers of prenatal tobacco smoke exposure: the correlation between serum and meconium and their association with infant birth weight. Environ Health [Internet]. 2010 Aug 27 [cited 2019 Mar 31];9(1):53. Available from: http://ehjournal.biomedcentral.com/articles/10.1186/1476-069X-9-53 [http://dx.doi.org/10.1186/1476-069X-9-53]

[35] Yarnall NJ, Hughes LM, Turnbull PS, Michaud M. Evaluating the effectiveness of the US Navy and Marine Corps Tobacco Policy: an assessment of secondhand smoke exposure in US Navy submariners. Tob Control 2013; 22(e1): e66-72.http://www.ncbi.nlm.nih.gov/ pubmed/22871902

[http://dx.doi.org/10.1136/tobaccocontrol-2012-050488] [PMID: 22871902]

[36] Martínez-Sánchez JM, Sureda X, Fu M, et al. Secondhand smoke exposure at home: assessment by biomarkers and airborne markers. Environ Res 2014; 133: 111-6.http://www.ncbi.nlm.nih.gov/ pubmed/24912142

[http://dx.doi.org/10.1016/j.envres.2014.05.013] [PMID: 24912142]

[37] Matsumoto A, Ichiba M, Payton NM, Oishi H, Hara M. Simultaneous measurement of urinary total nicotine and cotinine as biomarkers of active and passive smoking among Japanese individuals. Env Heal Prev Med [Internet]. 2013;18(3):244-50. Available from: http://www.ncbi.nlm. nih.gov/pubmed/23011941 [http://dx.doi.org/10.1007/s12199-012-0307-5]

[38] Hwang SH, Hwang JH, Moon JS, Lee DH. Environmental tobacco smoke and children's health. Korean J Pediatr 2012; 55(2): 35-41.https://www.ncbi.nlm.nih.gov/pubmed/22375147 [http://dx.doi.org/10.3345/kjp.2012.55.2.35] [PMID: 22375147]

[39] Wang Y, Yang M, Huang Z, Tian L, Niu L, Xiao S. Urinary cotinine concentrations in preschool children showed positive associations with smoking fathers. Acta Paediatr 2017; 106(1): 67-73.https://www.ncbi. nlm.nih.gov/pubmed/27748973

[http://dx.doi.org/10.1111/apa.13637] [PMID: 27748973]

[40] Martínez-Sánchez JM, González-Marrón A, Martín-Sánchez JC, Sureda X, Fu M, Pérez-Ortuño R, et al. Validity of self-reported intensity of exposure to second-hand smoke at home against environmental and personal markers. Gac Sanit 2017; 2017https://www.ncbi.nlm.nih.gov/pubmed/29102505 [PMID: 29102505]

[41] Jurado D, Muñoz C, Luna JdeD, Fernández-Crehuet M. Environmental tobacco smoke exposure in children: parental perception of smokiness at home and other factors associated with urinary cotinine in preschool children. J Expo Anal Environ Epidemiol 2004; 14(4): 330-6.https://www.ncbi.nlm.nih.gov/pubmed/15254480 [http://dx.doi.org/10.1038/sj.jea.7500329] [PMID: 15254480]

[42] Wilson T, Shamo F, Boynton K, Kiley J. The impact of Michigan's Dr Ron Davis smoke-free air law on levels of cotinine, tobacco-specific lung carcinogen and severity of self-reported respiratory symptoms among non-smoking bar employees. Tob Control 2012; 21(6): 593-5.https://www.ncbi.nlm.nih.gov/pubmed/22705599

[http://dx.doi.org/10.1136/tobaccocontrol-2011-050328] [PMID: 22705599]

[43] Matsumoto A, Ino T, Ohta M, Otani T, Hanada S, Sakuraoka A, et al. Enzyme-linked immunosorbent assay of nicotine metabolites. Env Heal Prev Med [Internet]. 2010/01/08. 2010;15(4):211-6. Available from: https://www.ncbi.nlm.nih.gov/pubmed/21432547

[http://dx.doi.org/10.1007/s12199-009-0129-2]

[44] Machado JdeB, Chatkin JM, Zimmer AR, Goulart AP, Thiesen FV. Cotinine and polycyclic aromatic hydrocarbons levels in the amniotic fluid and fetal cord at birth and in the urine from pregnant smokers. PLoS One 2014; 9(12)e116293http://www.ncbi.nlm.nih.gov /pubmed/25549364

[http://dx.doi.org/10.1371/journal.pone.0116293] [PMID: 25549364]

[45] Rubinstein ML, Shiffman S, Rait MA, Benowitz NL. Race, gender, and nicotine metabolism in adolescent smokers. Nicotine Tob Res [Internet]. 2012/12/13. 2013;15(7):1311-5. Available from: https://www.ncbi.nlm.nih.gov/pubmed/23239845

[http://dx.doi.org/10.1093/ntr/nts272]

[46] Davis RA, Stiles MF, deBethizy JD, Reynolds JH. Dietary nicotine: a source of urinary cotinine. Food Chem Toxicol 1991; 29(12): 821-7.http://www.ncbi.nlm.nih.gov/pubmed/1765327

[http://dx.doi.org/10.1016/0278-6915(91)90109-K] [PMID: 1765327]

[47] Dempsey D, Jacob P III, Benowitz NL. Nicotine metabolism and elimination kinetics in newborns. Clin Pharmacol Ther 2000; 67(5): 458-65.http://www.ncbi.nlm.nih.gov/pubmed/10824624 [http://dx.doi.org/10.1067/mcp.2000.106129] [PMID: 10824624]

[48] Molander L, Hansson A, Lunell E. Pharmacokinetics of nicotine in healthy elderly people. Clin Pharmacol Ther 2001; 69(1): 57-65.https://www.ncbi.nlm.nih.gov/pubmed/11180039

[http://dx.doi.org/10.1067/mcp.2001.113181] [PMID: 11180039]

[49] Dempsey D, Jacob P III, Benowitz NL. Accelerated metabolism of nicotine and cotinine in pregnant smokers. J Pharmacol Exp Ther 2002; 301(2): 594-8.http://www.ncbi.nlm.nih.gov/pubmed/11961061 [http://dx.doi.org/10.1124/jpet.301.2.594] [PMID: 11961061]

[50] Molander L, Hansson A, Lunell E, Alainentalo L, Hoffmann M, Larsson R. Pharmacokinetics of nicotine in kidney failure. Clin Pharmacol Ther 2000; 68(3): 250-60.https://www.ncbi.nlm.nih.gov/ pubmed/11014406

[http://dx.doi.org/10.1067/mcp.2000.109006] [PMID: 11014406]

[51] Chenoweth MJ, O'Loughlin J, Sylvestre MP, Tyndale RF. CYP2A6 slow nicotine metabolism is associated with increased quitting by adolescent smokers. Pharmacogenet Genomics 2013; 23(4): 232-5.https://www.ncbi.nlm.nih.gov/pubmed/23462429 [http://dx.doi.org/10.1097/FPC.0b013e32835f834d]

[PMID: 23462429]

[52] Yang M, Kunugita N, Kitagawa K, et al. Individual differences in 
urinary cotinine levels in Japanese smokers: relation to genetic polymorphism of drug-metabolizing enzymes. Cancer Epidemiol Biomarkers Prev 2001; 10(6): 589-93.

[PMID: 11401907]

[53] Nakajima M, Fukami T, Yamanaka $\mathrm{H}$, et al. Comprehensive evaluation of variability in nicotine metabolism and CYP2A6 polymorphic alleles in four ethnic populations. Clin Pharmacol Ther 2006; 80(3): 282-97.https://www.ncbi.nlm.nih.gov/pubmed/16952495 [http://dx.doi.org/10.1016/j.clpt.2006.05.012] [PMID: 16952495]

[54] Kim S. Overview of Cotinine Cutoff Values for Smoking Status Classification. Int J Environ Res Public Health 2016; 13(12): 1236.https://www.ncbi.nlm.nih.gov/pmc/articles/PMC5201377/ [http://dx.doi.org/10.3390/ijerph13121236] [PMID: 27983665]

\section{C) 2020 Oliva Fernandes etal.}

This is an open access article distributed under the terms of the Creative Commons Attribution 4.0 International Public License (CC-BY 4.0), a copy of which is available at: (https://creativecommons.org/licenses/by/4.0/legalcode). This license permits unrestricted use, distribution, and reproduction in any medium, provided the original author and source are credited. 